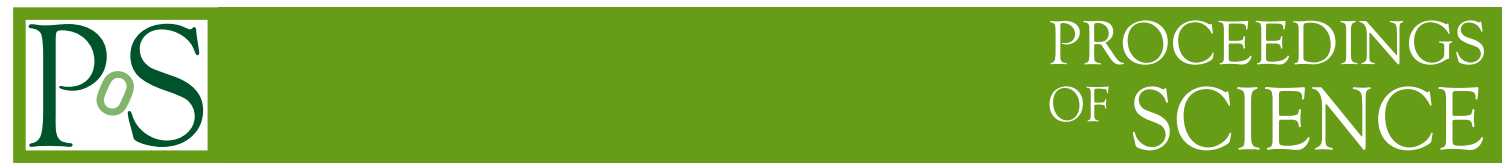

\title{
Diffractive and ultraperipheral physics with ALICE
}

\author{
Rainer SCHICKER ${ }^{* \dagger}$ \\ Ruprecht Karls University, Heidelberg \\ E-mail: schicker@physi.uni-heidelberg.de
}

The ALICE experiment consists of a central barrel, a muon spectrometer, zero degree calorimeters and additional detectors for trigger and event classification purposes. Such a geometry allows the implementation of a double gap trigger. A diffractive physics program of ALICE is outlined and a few specific reaction channels are discussed in order to illustrate the interest in such a diffractive physics program.

Physics at LHC 2008

29 September - October 4, 2008

Split, Croatia

\footnotetext{
${ }^{*}$ Speaker.

${ }^{\dagger}$ This work is supported in part by German BMBF under project 06HD197D
} 


\section{The ALICE Experiment}

The ALICE experiment is designed to take data in the high multiplicity environment of leadlead collisions[1]. The central barrel of ALICE and the forward muon spectrometer cover the pseudorapidity range $-0.9<\eta<0.9$ and $-4.0<\eta<-2.4$, respectively. Additional detectors for event classification and trigger purposes are located in the range $-4.0<\eta<5.0$.

\section{The ALICE Central Barrel}

The detectors in the ALICE central barrel track and identify hadrons, electrons and photons in the pseudorapidity range $-0.9<\eta<0.9$. The magnetic field strength of $0.5 \mathrm{~T}$ allows the measurement of tracks from very low transverse momenta of about $100 \mathrm{MeV} / \mathrm{c}$ to fairly high values of about $100 \mathrm{GeV} / \mathrm{c}$. The tracking detectors are designed to reconstruct secondary vertices resulting from decays of hyperons, D and B mesons. The main detector systems for these tasks are the Inner Tracking System (ITS), the Time Projection Chamber (TPC), the Transition Radiation Detector (TRD) and the Time of Flight array (TOF). These systems cover the full azimuthal angle within the pseudorapidity range $-0.9<\eta<0.9$. The Pixel Detector of the Inner Tracking System has an extended coverage of $-2.0<\eta<2.0$. Additional detectors with partial coverage of the central barrel are a PHOton Spectrometer (PHOS), an electromagnetic calorimeter (EMCAL) and a HighMomentum Particle Identification Detector (HMPID).

\section{The ALICE Zero Degree Neutron Calorimeter}

The Zero Degree Neutron Calorimeters (ZDC) are placed on both sides of the interaction point at a distance of $116 \mathrm{~m}$ [2]. The ZDC information can be used to select different diffractive topologies. Events of the types $p p \rightarrow p p X, p N^{*} X, N^{*} N^{*} X$ will have no signal, signal in one or in both of the ZDC calorimeters, respectively. Here, $X$ denotes a centrally produced diffractive state from which the diffractive L0 trigger is derived.

\section{The ALICE diffractive gap trigger}

Additional detectors for event classification and trigger purposes are located on both sides of the ALICE central barrel. First, an array of scintillator detectors (V0) is placed on both sides of the central barrel covering two units in pseudorapidity. Second, a Forward Multiplicity Detector (FMD) is located on both sides of the central barrel. The pseudorapidity coverage of this detector is $-3.4<\eta<-1.7$ and $1.7<\eta<5.1$, respectively. The geometry of the central barrel in conjunction with the additional detectors V0 and FMD is well suited for the definition of a rapidity gap trigger. Such a gap trigger can be defined by the requirement of signals coming from the central barrel detectors while V0 and FMD not showing any activity. This scheme requires a trigger signal from within the central barrel for L0 decision. The pixel detector of the ITS or the TOF array can deliver such a signal[3]. The integration of the TRD pretrigger system into the ALICE diffractive trigger scheme requires special considerations[4]. 


\section{ALICE diffractive physics}

The low luminosity of ALICE as compared to the other LHC experiments restricts the ALICE physics program to reactions with cross section of a few nb per unit of rapidity. Due to this low luminosity, the probability for multiple interactions within one bunch crossing is negligible. The tracking capabilities at very low transverse momenta together with the excellent particle identification make ALICE an unique facility to pursue a long term physics program of diffractive physics.

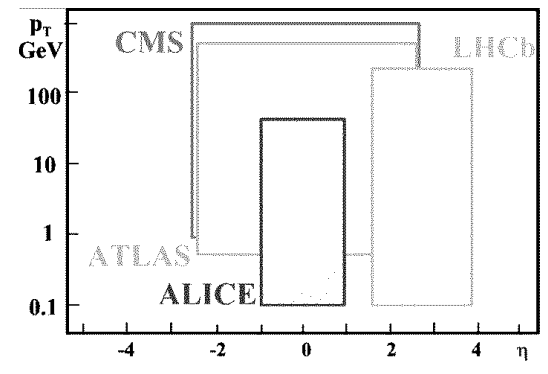

Figure 1: Rapidity and transverse momentum acceptance of the LHC experiments

Fig. 1 shows the transverse momentum acceptance of the four main LHC experiments. Not shown in this figure is the TOTEM experiment. The acceptance of the TOTEM telescopes is in the range of $3.1<|\eta|<4.7$ and $5.3<|\eta|<6.5$. The CMS transverse momentum acceptance of about $1 \mathrm{GeV} / \mathrm{c}$ shown in Fig. 1 represents a nominal value. The CMS analysis framework foresees the reconstruction of a few selected data samples to values as low as $0.2 \mathrm{GeV} / \mathrm{c}[5]$.

The ALICE gap trigger selects reactions which produce a rapidity interval void of particles. Such a signature can result from photon, W-boson or gluonic color singlet exchange. Two photon interactions are being discussed in the quest for new physics signals at the LHC[6]. The gluonic color singlet exchange has a C-even and C-odd part represented by the Pomeron and the Odderon.

Double pomeron exclusive reactions containing proton-antiproton as well as pion and kaon pairs have been studied at the Intersecting Storage Ring (ISR) at CERN. These events showed structures in the invariant pion pair mass which might be related to glueballs[7, 8]. At the SPS energy, data were taken in the central region and the azimuthal dependence of meson production was analyzed[9]. The ALICE gap trigger enables the continuation of such pomeron studies at the much higher energy of the LHC with correspondingly larger pomeron fluxes. The ALICE diffractive physics program includes the study of mesonic states such as glueballs, heavy quarkonia $\chi_{c}, \chi_{b}$ and the search for Odderon signatures in diffractive production of $J / \Psi[10]$. Glueball signatures can, for example, be searched for in the analysis of $\phi \phi$ events. The study of baryonic final states includes the analysis of $p \bar{p}$ pairs as discussed below as well as the search for hyperon pairs like $\Lambda \bar{\Lambda}$ and $\Sigma \bar{\Sigma}$.

\section{Central diffractive processes}

The formalism for central exclusive production of a system $\mathrm{X}$ folds the unintegrated gluon distribution with the elementary cross section $\mathrm{gg} \rightarrow \mathrm{X}$. Higher order QCD corrections to ensure no additional gluon radiation in the hard process are taken into account by the Sudakov suppression 
factor. The probability that the rapidity gap is not destroyed by additional soft particles from the rescattering of the protons is represented by the gap survival factor $\mathrm{S}^{2}$. Central exclusive production is thought to be a clean environment for identifying the Higgs boson at the LHC[11]. The calculated Higgs cross section is, however, small with large associated uncertainties due to the different ingredients as explained above. The cross section for exclusive production of photon-photon, jetjet and $\chi_{c}, \chi_{b}$ events can be calculated within the same formalism. In particular, the resulting cross section for $\chi_{c}$ and dijets is orders of magnitude higher than the Higgs cross section. A measurement of centrally produced $\chi_{c}$ and dijets is of interest in its own but also of importance for a systematic study of the uncertainties inherent in the formalism of central exclusive production.

\section{7. $\chi_{c}$ production}

A study of centrally produced $\chi_{c 0}$ in pp collisions at LHC energy $\sqrt{s}=14 \mathrm{TeV}$ was carried out in Regge formalism and perturbative QCD approach. This study finds cross section values of $\left.\frac{d \sigma}{d y}\right|_{y=0}=340 \mathrm{nb}$ and $30 \mathrm{nb}$ for exclusive and inclusive production, respectively[12]. Here, the exclusive cross section is larger as compared to the inclusive one due to the low mass of the $\chi_{c 0}$.

At the ALICE pp-luminosity $\mathscr{L}=5 \cdot 10^{30} \mathrm{~cm}^{-2} \mathrm{~s}^{-1}, 3.4 \cdot 10^{6} \chi_{c 0}$ 's are produced in $10^{6}$ seconds.

\begin{tabular}{|c|c|c|}
\hline decay mode & BR & signal \\
\hline$\chi_{c 0} \rightarrow \pi \pi$ & $7 \cdot 10^{-3}$ & $2.4 \cdot 10^{4}$ \\
$\chi_{c 0} \rightarrow K^{+} K^{-}$ & $6 \cdot 10^{-3}$ & $2.1 \cdot 10^{4}$ \\
$\chi_{c 0} \rightarrow \mathrm{J} / \Psi \gamma$ & $1 \cdot 10^{-2}$ & $3.5 \cdot 10^{4}$ \\
$\chi_{c 0} \rightarrow p \bar{p}$ & $2 \cdot 10^{-4}$ & 700 \\
\hline
\end{tabular}

Table 1: Branching ratios and signal sample of $\chi_{c 0}$ decays

Table 1 shows the branching ratio for different $\chi_{c 0}$ decay channels together with the signal sample resulting from $3.4 \cdot 10^{6}$ decays. The signal sample has to be folded with the detector acceptance and analysis efficiency for getting the number of reconstructed $\chi_{c 0}$ 's. A simulation study of the decay $\chi_{c 1,2} \rightarrow \mathrm{J} / \Psi \gamma$ finds an acceptance of $10 \%$ and a reconstruction efficiency of $0.9 \%$ for identifying the $\mathrm{J} / \Psi$ by its $\mathrm{e}^{+} \mathrm{e}^{-}$decay and the photon by the conversion method[13]. These values in conjunction with the $\chi_{c 0}$ cross section shown above results, however, in a data sample of very low statistics. Much better statistics can be achieved in the $\pi \pi, K K$ and $p \bar{p}$ decay channels. Of particular interest is the $p \bar{p}$ channel with a statistical uncertainty of a few percent while the background is thought to be much reduced as compared to the $\pi \pi$ and $K K$ channels.

\section{Exclusive vector meson production in lead-lead collisions}

The electromagnetic interaction between two nuclei can be studied in ultra-peripheral collisions where the impact parameter is larger than the sum of the two radii[14]. The photons of the electromagnetic field of the beams can interact in a variety of ways. First, two photon interactions will result in copious production of $\pi^{0}, \eta$ and $\eta^{\prime}$. Second, the photon of one beam can interact hadronically with a nucleus of the other beam resulting in photonuclear interactions. Inelastic and 
elastic photonuclear reactions are characterized by breakup and no-breakup of the nucleus, respectively. The elastic photonuclear cross section is dominated by exclusive vector meson production.

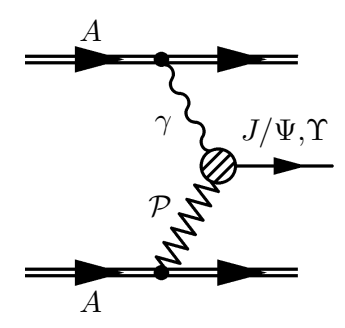

Figure 2: Exclusive vector meson production in AA collision

A photon can fluctuate into virtual vector meson states and subsequently get on-shell by diffractively scattering off a nucleus as depicted in Fig.2. The cross section for production of $\rho^{0}$, $\mathrm{J} / \Psi$ and $\Upsilon(1 \mathrm{~S})$ in lead-lead interactions at LHC energies is found to be $5.2 \mathrm{~b}, 32 \mathrm{mb}$ and $280 \mu \mathrm{b}$, respectively[15]. The detection of the $\rho$ by its $\pi^{+} \pi^{-}$and of the $\mathrm{J} / \Psi, \Upsilon$ by its $\mathrm{e}^{+} \mathrm{e}^{-}$decay results in data samples in one month of data taking of $2 \cdot 10^{8}, 1 \cdot 10^{5}$ and 400 , respectively.

\section{Conclusions}

A rapidity gap trigger in the ALICE experiment enables the measurement of many diffractive and electromagnetic reaction channels. These measurements open the way for a diverse diffractive physics program both in proton-proton and heavy-ion induced reactions.

\section{References}

[1] B. Alessandro et al, ALICE Collaboration, J. Phys. G 32 (2006) 1295

[2] R. Arnaldi et al, Nucl. Instr. and Meth. A 564 (2006) 235

[3] The ALICE coll., K. Aamodt et al, The ALICE experiment at the LHC, (2008) JINST_3_S08002

[4] R. Schicker, Nucl. Phys. B 179-180 (2008) 196

[5] D. d'Enterria et al, Addendum CMS technical design report, J. Phys. G 34 (2007) 2307

[6] K. Piotrzkowski, Nucl. Phys. B 179-180 (2008) 2

[7] T. Åkesson et al, Nucl. Phys. B 264 (1986) 154

[8] A. Breakstone et al, Z. Phys. C 48 (1990) 569

[9] F. Close et al, Phys. Lett. B 477 (2000) 13

[10] R. Schicker, Proceedings HERA-LHC workshop (2008), arXiv:0812.2176

[11] A. De Roeck et al, Eur. Phys. J. C25 (2002) 391

[12] V.A. Khoze et al, Eur. Phys. J. C35 (2004) 211

[13] P. Gonzalez et al, arXiv:0811.1592

[14] K. Hencken et al, Phys. Rept. 458 (2008) 1

[15] S. Klein et al, Phys. Rev. Lett. 92 (2004) 142003 